\title{
Knockdown of myosin VI by lentivirus-mediated short hairpin RNA suppresses proliferation of melanoma
}

\author{
HUI LI*, FUSHENG ZHOU*, HONGYAN WANG, DA LIN, GANG CHEN, \\ XIANBO ZUO, LIANGDAN SUN, XUEJUN ZHANG and SEN YANG \\ Department of Dermatology, Institute of Dermatology, The First Affiliated Hospital, \\ Anhui Medical University, Hefei, Anhui 230032, P.R. China
}

Received June 24, 2014; Accepted March 6, 2015

DOI: $10.3892 / \mathrm{mmr} .2015 .4261$

\begin{abstract}
Myosin VI has been reported to be associated with the progression of ovarian and prostate cancer. The aim of the present study was to reveal the role of myosin VI in the proliferation of melanoma. Briefly, lentivirus-mediated short hairpin RNA (shRNA) was designed specifically to silence myosin VI in A375 and A431 cell lines. Expression levels of myosin VI were then analyzed in the two cell lines by quantitative polymerase chain reaction and western blot analyses. Cell viability was assessed using MTT and colony formation assays. In addition, the cell cycle distribution was determined by flow cytometry. The results demonstrated that knockdown of myosin VI significantly suppressed melanoma cell viability and proliferation, and induced cell cycle arrest in G0/G1 phase. To the best of our knowledge, the present study was the first to assess the role of myosin VI in the growth of melanoma. Knowledge of the underlying mechanism of the role myosin VI in skin cancer cells may aid in the development of novel methods of melanoma diagnosis and therapy in the future.
\end{abstract}

\section{Introduction}

Cutaneous melanoma is the most common malignant neoplasm of human skin, with the number of cases increasing (1). An estimated 76,690 novel cases of melanoma occurred in the USA in 2013 (2). Conventional treatment is commonly performed through tumorectomy along with resection of lymph nodes in the neighboring area. Although the five-year survival rate was improved, it has remained as low as $70 \%$

Correspondence to: Dr Sen Yang, Department of Dermatology, Institute of Dermatology, The First Affiliated Hospital, Anhui Medical University, 218 Jixi Road, Hefei, Anhui 230032, P.R. China E-mail: yangsendr@163.com

*Contributed equally

Key words: cell cycle arrest, melanoma, myosin VI, proliferation, RNA interference in African Americans (3). Other treatments, including chemotherapy and radiotherapy, have been proved to be applicable and are widely adopted, but serious side-effects cannot be avoided (4,5). Therefore, a novel therapy against skin cancer with better prognostics is required.

Shiu et al (6) reported that the survival rate of pregnant patients and parous women was significantly low compared with that of nulliparous patients with stage II melanoma. This observation indicated that the effect of cell endocrine in melanoma is closely associated with exocytosis (7), which in turn is regulated by myosin VI. Myosin VI is an unconventional motor protein, which moves towards the minus end of actin filaments. It manipulates a wide range of cellular processes, including exocytosis, cell migration, cell division, endocytosis and cytokinesis $(8,9)$. Myosin VI interacts with specific adaptor proteins and is regulated by multiple regulatory signals and modifications, including actin filament geometry, which determines the myosin VI binding sites (10), and disturbance or block of these cellular signaling pathways leads to the development of diseases, including deafness and cancer (8).

Several studies have found abnormal expression of myosin VI in ovary and prostate cancer; however, the underlying mechanism and the association and of myosin VI and human cancer remained poorly understood until Yoshida et al (11) discovered the overexpression of myosin IV in ovary carcinoma, but not in normal ovaries. Through inhibition of myosin VI expression, the spreading and migration of high-grade ovary carcinoma cells was impeded in vitro. Wei et al (12) found that the expression levels of myosin IV were elevated in the Golgi apparatus in prostate cancer, but not in normal cells. Furthermore, Dunn et al (13) impaired the migration and colony formation in vitro by silencing myosin VI in prostate cancer cells. All of these findings suggested that myosin VI is involved in tumor invasion and may be a diagnosis biomarker and therapeutic target of ovary and prostate cancer.

In spite of the studies available on prostate and ovary cancer, the specific effect of myosin VI on skin cancer cell viability has remained to be clearly demonstrated. In the present study, the role of myosin VI in melanoma was firstly studied. Specific knockdown of myosin VI was performed by RNA interference (RNAi), and its effect on the proliferation, colony formation and cell cycle of the melanoma cell lines 
A375 and A431 was investigated. Knockdown of myosin VI may be a novel therapeutic method for melanoma in future, which may be the key to fundamentally cure cancer with little side-effects by regulating the tagged gene.

\section{Materials and methods}

Cell culture. The melanocytes (A375 and A431) and human embryonic kidney cells (293T) were obtained from the Cell Bank of Chinese Academy of Sciences (Shanghai, China). All cell lines were cultured with the Dulbecco's Modified Eagle's Medium (Hyclone, Logan, UT, USA) with 10\% FBS (Gibco Life Technologies, Carlsbad, CA, USA) at $37^{\circ} \mathrm{C}$ in an incubator with humidified air and $5 \% \mathrm{CO}_{2}$.

Lentiviral vector design and construction. Two lentiviral vectors were designed to knock down the myosin VI gene (NM_004999) and to avoid the non-specific knockdown effect, respectively. The sequence of the shRNA designed to silence myosin VI was 5'-GTGAATCCAGAGATAAGTTTACTCGAG TAAACTTATCTCTGGATTCACTTTTT-3', and that of the control siRNA was 5'-TTCTCCGAACGTGTCACGT-3'. The stem-loop-stem oligos (shRNAs) were synthesized, annealed and ligated into the Nhe I/Pac I-linearized $\mathrm{pFH}-\mathrm{L}$ vector (Shanghai Hollybio, Shanghai, China). After confirmation of the vectors by nucleotide sequencing, the successfully constructed vectors, packaged by pVSVG-I and pCMV $\Delta$ R8.92 plasmids (Shanghai Hollybio), were transfected into $293 \mathrm{~T}$ cells to avail larger quantities of usable lentivirus, which were subsequently harvested $72 \mathrm{~h}$ after transfection and then purified.

Lentivirus infection and gene knockdown. A375 and A431 cells $\left(5 \times 10^{4}\right.$ cells/well) were seeded in six-well plates and infected with MYO6 shRNA lentivirus (Lv-shMYO6) or control shRNA lentivirus (Lv-shCon) at a multiplicity of infection of 20 or 25, respectively, supplemented with polybrene (Santa Cruz Biotechnology, Inc., Dallas, TX, USA). Purification was conducted as previously described (14). A BX50 fluorescent microscope (Olympus Corporation, Tokyo, Japan) was then used to observe infection efficiency $96 \mathrm{~h}$ post-infection.

Quantitative polymerase chain reaction $(q P C R)$. $\mathrm{qPCR}$ was performed on a Bio-Rad MyCycler Connect Real-Time PCR platform (Bio-Rad Laboratories, Hercules, CA, USA). Total RNA was extracted from the A375 and A431 cells with TRIzol ${ }^{\circledR}$ (Invitrogen Life Technologies, Carlsbad, CA, USA). The RNA ( $2 \mu \mathrm{g})$ was then reverse transcribed using an M-MLV Reverse Transcriptase kit (Promega, Madison, WI, USA) according to the manufacturer's instructions. Moloy murine leukemia virus reverse transcriptase (cat no. M1705; Promega) was used as the reverse transcriptase in this method. The qPCR reaction system consisted of $2 \mathrm{X}$ SYBR premix ex $\operatorname{taq}(10 \mu \mathrm{l})$, forward and reverse primers $(2.5 \mu \mathrm{M} ; 0.8 \mu \mathrm{l})$, cDNA $(5 \mu \mathrm{l})$ and $\mathrm{ddH}_{2} \mathrm{O}(4.2 \mu \mathrm{l})$. For actin, the forward primer was 5'-GTGGACATCCGCAAAGAC-3' and the reverse primer was 5'-AAAGGGTGTAACGCAACTA-3'. For myosin VI, the forward primerwas 5'-AATCACTGGCTCACATGCAG-3' and the reverse primer was 5'-AATGCGAGGTTTGTGTCTCC-3'. All primers were obtained from 3D-HTS Company
(Shanghai, China). The qPCR program was as follows: Initial denaturation at $95^{\circ} \mathrm{C}$ for $1 \mathrm{~min}, 40$ cycles of denaturation at $95^{\circ} \mathrm{C}$ for $5 \mathrm{sec}$ and annealing extension at $60^{\circ} \mathrm{C}$ for $20 \mathrm{sec}$. The cycle threshold $(\mathrm{Ct})$ normalized to that of actin was used to determine the relative expression of myosin VI using the $2^{-\Delta \Delta \mathrm{Ct}}$ method.

Western blot analysis. Lentivirus-transduced cells were lysed in 2X SDS sample buffer (100 mM Tris- $\mathrm{HCl}$ (pH 6.8), $10 \mathrm{mM}$ EDTA, 4\% SDS and 10\% glycine; Sigma-Aldrich, St. Louis, MO, USA). Proteins were separated by $10 \%$ SDS-PAGE (Sigma-Aldrich). In each lane of the gels, $30 \mu \mathrm{g}$ of protein was added and electrophoresis was performed under $50 \mathrm{~V}$ for $3 \mathrm{~h}$. The transmembrane procedure was performed under $300 \mathrm{~mA}$ for $1.5 \mathrm{~h}$. Next, the membrane was incubated with rabbit monoclonal anti-MYO6 (1:500; cat. no. M0691; Sigma-Aldrich) and mouse polyclonal anti-GADPH (1:3,000; cat. no. 10494-1-AP; Proteintech Group, Inc., Chicago, IL, USA) primary antibodies at $4^{\circ} \mathrm{C}$ overnight, and horseradish peroxidase-conjugated goat anti-mouse/rabbit IgG secondary antibodies (cat. no. sc-2054; Santa Cruz Biotechnology, Inc.) at room temperature for $2 \mathrm{~h}$. GAPDH protein was used as a loading control.

MTT assay. Following lentiviral infection, A375 $\left(2 \times 10^{3}\right.$ cells/well) and A431 ( $3 \times 10^{3}$ cells/well) were seeded into 96-well plates, respectively. The number of cells was measured at time-points indicated in the figures. $20 \mu \mathrm{l} \mathrm{MTT}$ solution $(5 \mathrm{mg} / \mathrm{ml})$ was added into each well. MTT solution was removed by aspiration following incubation, and $100 \mu \mathrm{l}$ acidic isopropanol (10\% SDS, 5\% isopropanol and $0.01 \mathrm{~mol} / 1$ $\mathrm{HCl}$; Sigma-Aldrich) was added. The absorbance of each plate was measured at $595 \mathrm{~nm}$ using an ELx800 spectrophotometer (BioTek Instruments, Inc., Winooski, VT, USA).

Colony formation assay. Following lentiviral infection, A375 cells were seeded into six-well plates at a concentration of 600 cells/well and maintained at $37^{\circ} \mathrm{C}$ for seven days. The culture media were changed every 2-3 days. When the colonies were formed, the plate was washed and fixed with 4\% paraformaldehyde (Sangon Biotech Co., Ltd., Shanghai, China), stained with crystal violet (Beyotime Institute of Biotechnology, Shanghai, China) for $20 \mathrm{~min}$ and washed three times with $\mathrm{ddH}_{2} \mathrm{O}$. The number of colonies (defined as $>50$ cells/colony) was counted.

Flow cytometric analysis. Following lentiviral infection, A375 cells were seeded into $6-\mathrm{cm}$ dishes at a concentration of $5 \times 10^{4}$ cells/dish. Cells were harvested following trypsinization, washed with phosphate-buffered saline (PBS) and fixed in $80 \%$ ethanol (Sigma-Aldrich) at $-20^{\circ} \mathrm{C}$ for $24 \mathrm{~h}$. Cells were then collected by centrifugation at $1,300 \mathrm{x} \mathrm{g}$ for $5 \mathrm{~min}$, resuspended in the staining solution $(100 \mu \mathrm{g} / \mathrm{ml}$ RNase A and $50 \mu \mathrm{g} / \mathrm{ml}$ propidium iodide in PBS), and incubated for $1 \mathrm{~h}$ at $37^{\circ} \mathrm{C}$. The stained cells were subjected to flow cytometric analysis using a FACSCalibur II flow cytometer and Cell Quest Pro v. 3.3 (BD Biosciences, San Jose, CA, USA).

Statistical analysis. Values are expressed as the mean \pm standard deviation (SD) of at least three independent experiments 
A


$\mathbf{A 4 3 1}$
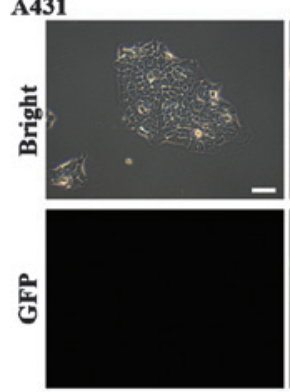

Lv-shCon
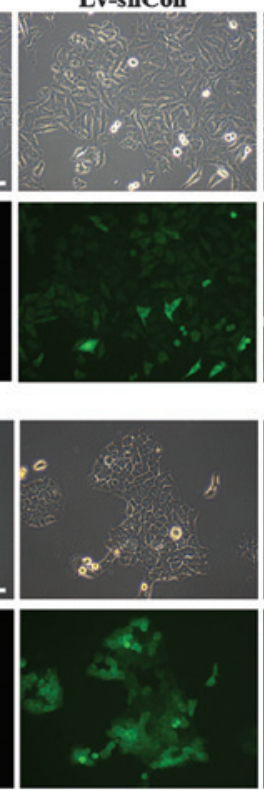

Lv-shMYO6
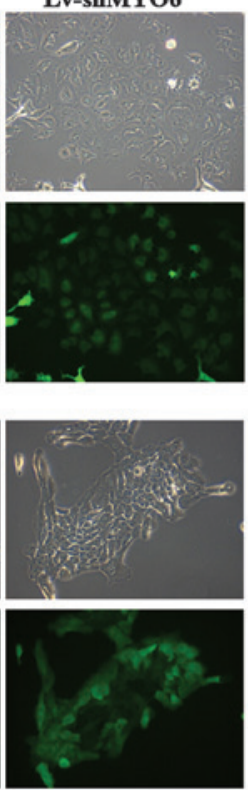

B
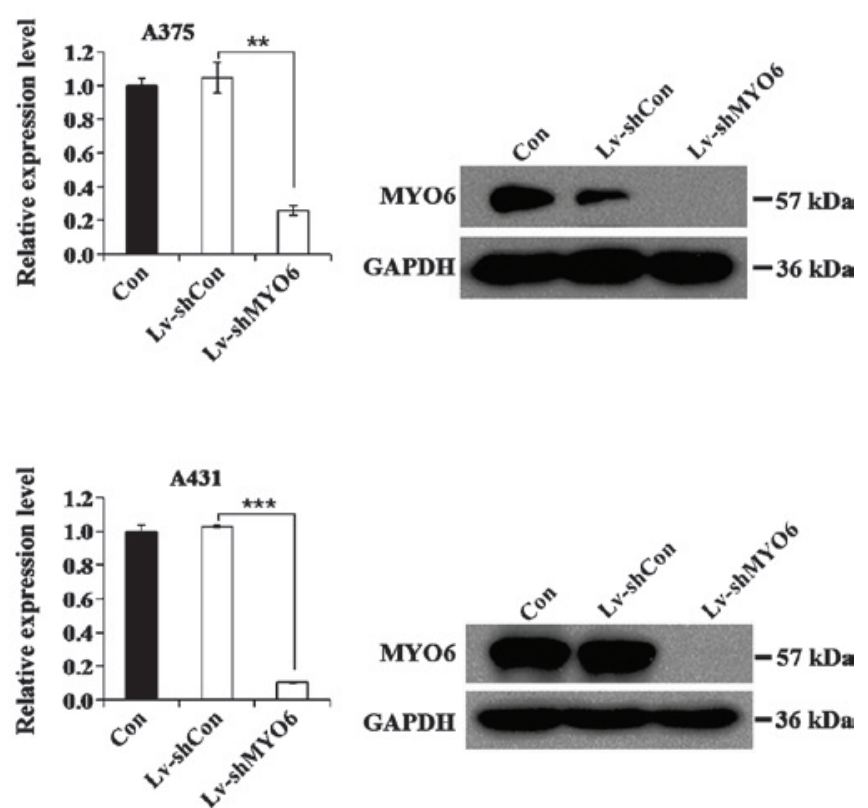

Figure 1. Lentivirus infection efficiency and knockdown efficiency in melanoma cells. Bright field and fluorescence photomicrographs after transduction of (A) A375 and A431 cells in Con, Lv-shCon and Lv-shMYO6 groups, respectively (scale bar, $100 \mu \mathrm{m}$ ). (B) Relative mRNA expression levels of myosin VI in A431 and A431 cells by small interfering RNA assessed using quantitative polymerase chain reaction (bar graphs) and protein levels assessed using western blot analysis (blots). The data are presented as the mean \pm standard deviation of three independent experiments. ${ }^{* *} \mathrm{P}<0.01$, $^{* * *} \mathrm{P}<0.001$, compared with $\mathrm{Lv}$-shCon. Con, control; LV, lentivirus, GFP, green fluorescent protein; sh, small hairpin; myo, myosin.

A

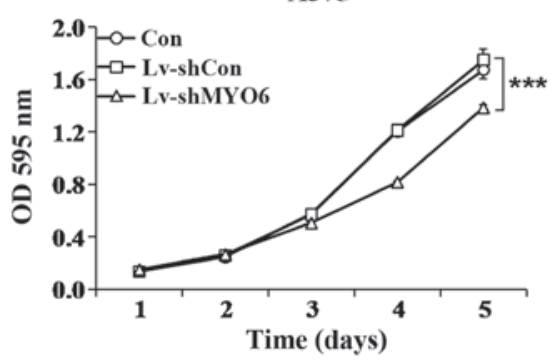

B



Figure 2. Viability of melanoma cells following myosin VI silencing. OD at $595 \mathrm{~nm}$ of (A) A375 and (B) A431 cells in Con, Lv-shCon and Lv-shMYO6 groups respectively. The data are presented as the mean \pm standard deviation of three independent experiments. ${ }^{* * * *} \mathrm{P}<0.001$, compared to LV-shCon. OD, optical density; Con, control; LV, lentivirus, sh, small hairpin; myo, myosin.

performed in triplicate. Statistical significance was assessed with Student's t-test. All statistical analyses were performed using Prism 5 for Windows (GraphPad Software, Inc., La Jolla, CA, USA). $\mathrm{P}<0.05$ was considered to indicate a statistically significant difference between values.

\section{Results}

Myosin VI knockdown by lentivirus-mediated RNAi in melanoma cells. A specific lentivirus-shRNA system (Lv-shMYO6) was designed to knockdown myosin VI, and was then used to investigate whether myosin VI expression was required for the growth and viability of human melanoma cells. In addition, a control shRNA (Lv-shCon) was constructed to avoid the non-specific gene-silencing effect of the lentivirus alone $(15,16)$. Either of the two lentivirus systems was transfected into A375 and A431 cells. The efficiency of Lv-shMYO6 transfection was observed under a fluorescent microscope, and $>80 \%$ of cells expressed GFP, whereas the non-infected control group showed no green fluorescence at all (Fig. 1A), which suggested that the efficiency of lentiviral infection was stable and substantial.

qPCR and western blot analyses were performed to evaluate the knockdown efficiency five days after infection. There was no significant difference between the expression of myosin VI in the Lv-shCon group and that in the control (Con) group in the two cell lines. However, the expression of myosin VI was reduced by $75.0 \%$ in A375 cells $(\mathrm{P}<0.01$; Fig. 1B, top) and $89.9 \%$ in A431 cells $(\mathrm{P}<0.001$; Fig. 1B, bottom), respectively, compared to that in the Lv-shCon group. These results showed that the expression of myosin VI was effectively and specifically switched off by the lentivirus system in the two cell lines. Therefore, Lv-shMYO6 was able to be safely used as a downregulator to examine the role of the myosin VI gene in human skin cancer cells. 


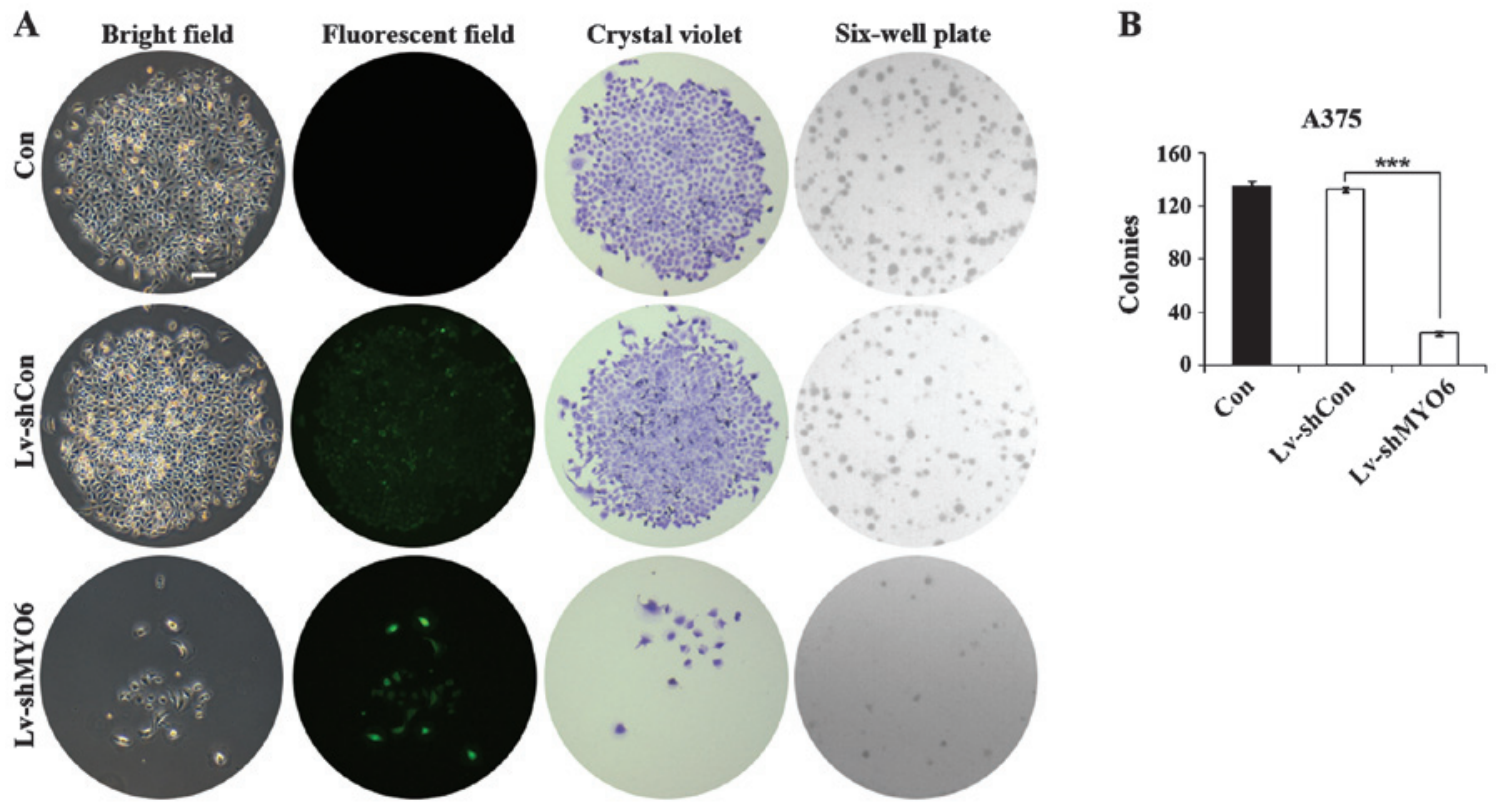

Figure 3. Colony formation ability of A375 cells following myosin VI silencing. (A) Colony formation of A375 cells in Con, Lv-shCon and Lv-shMYO6 groups Cells were visualized by bright field or fluorescent field microscopy, following crystal violet staining or in a six-well plate, respectively (scale bar, $250 \mu \mathrm{m}$; magnification, x40). (B) Statistical evaluation of colony numbers in Con, Lv-shCon and Lv-shMYO6 groups. The data are presented as the mean \pm standard deviation of three independent experiments. ${ }^{* * *} \mathrm{P}<0.001$, compared to Lv-shCon. Con, control; LV, lentivirus, sh, small hairpin; myo, myosin.
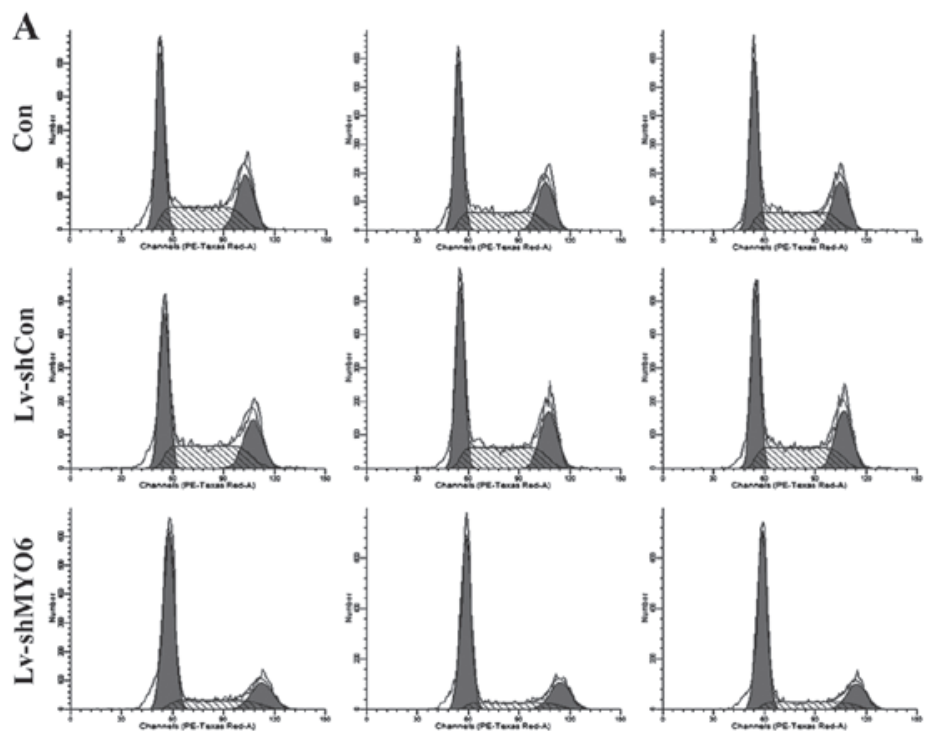

B

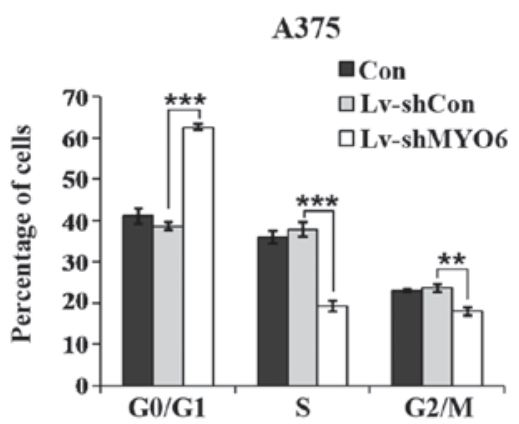

Figure 4. Myosin VI silencing caused cell cycle arrest. (A) Cell cycle analysis of A375 cells by flow cytometry in Con, Lv-shCon and Lv-shMYO6 groups respectively. (B) Quantification of percentage of cells at various stages of the cell cycle. The data are presented as the mean \pm standard deviation of three independent experiments. ${ }^{* * *} \mathrm{P}<0.01,{ }^{* * *} \mathrm{P}<0.001$, compared to Lv-shCon. Con, control; LV, lentivirus, sh, small hairpin; myo, myosin.

Knockdown of myosin VI decreases cell viability and growth. The populations of A375 and A431 cells in all groups were routinely observed in vitro through MTT assays at indicated time-points following infection (Fig. 2). The viability in the Lv-shMYO6 group was significantly decreased compared with that in the Con and Lv-shCon groups, whereas the Con and Lv-shCon exhibited similar viability. In more detail, on day four, the optical density (OD) value at $595 \mathrm{~nm}$ of A375 cells in the Lv-shMYO6 group $(0.815 \pm 0.014)$ was lower than that in the Lv-shCon group $(1.211 \pm 0.030 ; \mathrm{P}<0.001)$ and the Con group $(1.203 \pm 0.027 ; \mathrm{P}<0.001)$. On day five, the OD value at $595 \mathrm{~nm}$ of A375 cells in the LV-shMYO6 group $(1.382 \pm 0.025)$ was markedly lower than that in the Lv-shCon group (1.747 \pm 0.084 ; $\mathrm{P}<0.001)$ and the Con group $(1.672 \pm 0.065 ; \mathrm{P}<0.001)$. The population gap between the Lv-shMYO6 and Con groups remained distinctive. The effect on the viability of A431 cells was more marked than that on the viability of A375 cells. The population of A431 cells in the Lv-shMYO6 group was $48.1 \%$ of that in the Con group on day five $(\mathrm{P}<0.001)$. Therefore, it is reasonable to conclude that lentivirus-mediated myosin VI silencing had a suppressive effect on the viability and proliferation of melanoma cells. 
In addition, the proliferation of A375 cells was determined by colony formation assay. As shown in the bright field, fluorescent field and crystal violet-stained microscopy images in Fig. 3A, the size of a single colony in the Lv-shMYO6 group was significantly smaller compared with that in the Con and Lv-shCon groups. In addition, the number of colonies in the Lv-shMYO6 group was lower compared with that in the Con and Lv-shCon groups, as shown in the image of the six-well plate experiment. As shown in Fig. 3B, compared with that in the Lv-shCon group, the number of colonies was decreased by $82.0 \%$ in the Lv-shMYO6 group $(\mathrm{P}<0.001)$, while the colony numbers in the Con group had were similar to those in the Lv-shCon group. The significant decrease in the proliferation of the two cell lines indicated that knockdown of myosin VI impeded the growth of melanoma, which further suggested that myosin VI may be a potential therapeutic target of melanoma.

MYO6 siliencing causes G0/G1 phase arrest. The cell cycle distribution of A375 cells following myosin VI silencing was analyzed by flow cytometric analysis (17). As illustrated in Fig. 4A, myosin VI silencing significantly altered the cell cycle distribution, with the G2/M-phase population significantly decreased in the LV-shMYO6 group in compared with that in the Con and Lv-shCon groups. Quantification of the cell cycle distributions (Fig. 4B) revealed that the S-phase

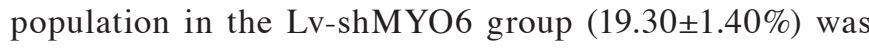
markedly decreased compared with that in the Lv-shCon group $(37.74 \pm 1.76 \%$; $\mathrm{P}<0.001)$. Furthermore, the G2/M-phase population in the Lv-shMYO6 group $(18.11 \pm 0.95 \%)$ was decreased compared with that in the Lv-shCon group (23.63 $\pm 0.96 \%$; $\mathrm{P}<0.01)$. However, the G0/G1-phase popula-

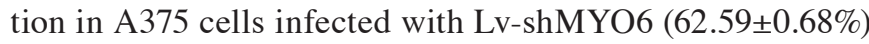
was markedly increased compared with that in the Con $(40.99 \pm 0.10 \% ; \mathrm{P}<0.001)$ and Lv-shCon $(38.62 \pm 0.87 \%$; $\mathrm{P}<0.001)$ groups. These results suggested that the impaired cell proliferation and colony formation may have been caused by cell cycle arrest in G0/G1 phase. Cells may have been hindered from entering $\mathrm{S}$ and $\mathrm{G} 2 / \mathrm{M}$ phases, so that they were no longer able to divide, which led to a decrease in their growth.

\section{Discussion}

Melanoma is one of the most fatal types of cancer (1). In spite of the development of therapeutic protocols, the survival rate of colon cancer patients has remained low due to its high potential to form metastasis. As a large number of tumor-inducing mutations and differentially expressed genes have been identified in melanoma, it is important to identify novel molecular targets for the development of novel treatments and biomarkers (4).

Myosin VI is a major gene which demonstrates cancer-specific overexpression, and is closely associated with membrane trafficking, clathrin-mediated endocytosis, exocytosis at the Golgi complex and cell migration (18-20). Numerous studies have been performed to reveal the structure, function and regulatory mechanisms of myosin VI, yet the association between myosin VI and cancer was only appropriately emphasized at the beginning of this century $(21,22)$. It was found that myosin VI is overexpressed, particularly at late stages of ovary and prostate cancer, but not in normal tissue; therefore, myosin VI is a biomarker for ovary cancer and prostate cancer $(11,12)$. A subsequent study showed that silencing myosin VI had an inhibiting effect on cell colony formation and migration of malignant cancer cells (13). These results inspired us to investigate whether knockdown of myosin VI exhibited any anti-melanoma effect. To the best our knowledge, the present study was the first to investigate the role of myosin VI in melanoma. Lentivirus-based shRNA expression systems were introduced to silence myosin VI in two different melanoma cell lines (A375 and A431) in order to investigate whether myosin VI is a potential therapeutic target of melanoma. The results indicated that the constructed lentivirus-mediated knockdown system was effective in silencing myosin VI at the gene transcription as well as the protein level. Following the specific knockdown of myosin VI, the viability of melanoma cell lines was significantly suppressed. These results suggested that myosin VI has an essential role in melanoma growth and migration. Further study of the cell cycle using flow cytometry was performed in order to investigate the underlying mechanism of the decrease of the cell proliferation rate following myosin VI knockdown. It was found that following myosin VI knockdown, the cell population in G0/G1 was increased. Simultaneously, the cell populations in $\mathrm{S}$ phase and $\mathrm{G} 2 / \mathrm{M}$ phase were concomitantly reduced compared with those in the control groups. This implied that the cell cycle was arrested at the G0/G1 following myosin VI silencing. With a lower amount of cells entering $\mathrm{S}$ and $\mathrm{G} 2 / \mathrm{M}$ phase, DNA replication and cell mitosis were impeded, which explains for the decreased cell proliferation and viability observed following myosin VI silencing.

In spite of the lack of extensive knowledge of the underlying mechanism and role of myosin VI in carcinogenesis as well as the anti-cancer effect of its knockdown, previous studies have provided three clues: Firstly, it has been reported that myosin VI interacted with GIPC1 to form a protein complex. GIPC1 regulates trophoblast glycoprotein, which is a prognostic factor in colorectal, ovarian and gastric cancer (23), tyrosinase-related protein 1, which affects melanocyte proliferation and melanocyte cell death (24), and T6BP, which is a binding partner of myosin VI involved in cell adhesion (25). Secondly, myosin VI binds to tumor suppressor gene DAB2, leading to the downregulation of DAB2 in ovarian carcinoma cell lines (26). Finally, myosin VI is a mediator of the p53-dependent cell survival pathway (27), while p53 is recognized as a cancer suppressor gene. These findings are a foundation for further study on the role of myosin VI in cancer.

In conclusion, the present study was the first to reveal that myosin VI is significantly correlated with melanoma. A specific myosin VI-targeting lentivirus system was then constructed to successfully knock down this gene. It was found that silencing myosin VI markedly inhibit colony formation and cell viability along with G0/G1 phase arrest. The results of the present study, supported by previous studies of myosin VI in ovary and prostate cancer (11-13), indicated that myosin VI is a prospective biomarker of various types of cancer and a promising therapeutic target for melanoma. 


\section{References}

1. Bittner M, Meltzer P, Chen Y, et al: Molecular classification of cutaneous malignant melanoma by gene expression profiling. Nature 406: 536-540, 2000.

2. Siegel R, Naishadham D and Jemal A: Cancer statistics, 2013. CA Cancer J Clin 63: 11-30, 2013.

3. DeSantis C, Naishadham D and Jemal A: Cancer statistics for African Americans, 2013. CA Cancer J Clin 63: 151-166, 2013.

4. Bajetta E, Del Vecchio M, Bernard-Marty C, et al: Metastatic melanoma: Chemotherapy. Semin Oncol 29: 427-445, 2002.

5. Overgaard J: The role of radiotherapy in recurrent and metastatic malignant melanoma: a clinical radiobiological study. Int J Radiat Oncol Biol Phys 12: 867-872, 1986.

6. Shiu MH, Schottenfeld D, Maclean B and Fortner JG: Adverse effect of pregnancy on melanoma: a reappraisal. Cancer 37: 181-187, 1976.

7. Dolensek J, Skelin M and Rupnik MS: Calcium dependencies of regulated exocytosis in different endocrine cells. Physiol Res 60 (Suppl 1): 29-38, 2011.

8. Buss F and Kendrick-Jones J: How are the cellular functions of myosin VI regulated within the cell? Biochem Biophys Res Commun 369: 165-175, 2008.

9. Wells AL, Lin AW, Chen LQ, et al: Myosin VI is an actin-based motor that moves backwards. Nature 401: 505-508, 1999.

10. Rock RS, Rice SE, Wells AL, Purcell TJ, Spudich JA and Sweeney HL: Myosin VI is a processive motor with a large step size. Proc Natl Acad Sci USA 98: 13655-13659, 2001.

11. Yoshida H, Cheng W, Hung J, et al: Lessons from border cell migration in the drosophila ovary: A role for myosin VI in dissemination of human ovarian cancer. Proc Natl Acad Sci USA 101: 8144-8149, 2004.

12. Wei S, Dunn TA, Isaacs WB, De Marzo AM and Luo J: GOLPH2 and MYO6: putative prostate cancer markers localized to the Golgi apparatus. Prostate 68: 1387-1395, 2008.

13. Dunn TA, Chen S, Faith DA, et al: A novel role of myosin VI in human prostate cancer. Am J Pathol 169: 1843-1854, 2006.

14. ter Brake O, Konstantinova P, Ceylan M and Berkhout B Silencing of HIV-1 with RNA interference: A multiple shRNA approach. Mol Ther 14: 883-892, 2006.
15. Bridge AJ, Pebernard S, Ducraux A, Nicoulaz AL and Iggo R: Induction of an interferon response by RNAi vectors in mammalian cells. Nat Genet 34: 263-264, 2003.

16. Samuel CE: Knockdown by RNAi-proceed with caution. Nat Biotechnol 22: 280-282, 2004.

17. Pozarowski P and Darzynkiewicz Z: Analysis of cell cycle by flow cytometry. Methods Mol Biol 281: 301-311, 2004.

18. Buss F, Arden SD, Lindsay M, Luzio JP and Kendrick-Jones J: Myosin VI isoform localized to clathrin-coated vesicles with a role in clathrin-mediated endocytosis. EMBO J 20: 3676-3684, 2001.

19. Sahlender DA, Roberts RC, Arden SD, et al: Optineurin links myosin VI to the Golgi complex and is involved in Golgi organization and exocytosis. J Cell Biol 169: 285-295, 2005.

20. Morris SM, Arden SD, Roberts RC, et al: Myosin VI binds to and localises with Dab2, potentially linking receptor-mediated endocytosis and the actin cytoskeleton. Traffic 3: 331-341, 2002.

21. Sakakura C, Hagiwara A, Nakanishi M, et al: Differential gene expression profiles of gastric cancer cells established from primary tumour and malignant ascites. Br J Cancer 87: 1153-1161, 2002.

22. Geisbrecht ER and Montell DJ: Myosin VI is required for E-cadherin-mediated border cell migration. Nat Cell Biol 4: 616-620, 2002.

23. Awan A, Lucic MR, Shaw DM, et al: 5T4 interacts with TIP-2/GIPC, a PDZ protein, with implications for metastasis. Biochem Biophys Res Commun 290: 1030-1036, 2002.

24. Sarangarajan R and Boissy RE: Tyrp1 and oculocutaneous albinism type 3. Pigment Cell Res 14: 437-444, 2001.

25. Morriswood B, Ryzhakov G, Puri C, et al: T6BP and NDP52 are myosin VI binding partners with potential roles in cytokine signalling and cell adhesion. J Cell Sci 120: 2574-2585, 2007.

26. Mok SC, Chan WY, Wong KK, et al: DOC-2, a candidate tumor suppressor gene in human epithelial ovarian cancer. Oncogene 16: 2381-2387, 1998.

27. Jung EJ, Liu G, Zhou WJ and Chen XB: Myosin VI is a mediator of the p53-dependent cell survival pathway. Mol Cell Biol 26: 2175-2186, 2006 